\title{
High PANX1 Expression Leads To Neutrophil Recruitment And Formation of A High Adenosine Immunosuppressive Tumor Microenvironment In Triple-Negative Breast Cancer
}

\section{Wuzhen Chen}

Department of Breast Surgery (Surgical Oncology)

\section{Baizhou Li}

Zhejiang University School of Medicine

\section{Chao Ni}

Department of Breast Surgery (Surgical Oncology)

\section{Fang Jia}

Department of Breast Surgery (Surgical Oncology)

Jiaxin Li

Department of Breast Surgery (Surgical Oncology)

Huanhuan Huang

Department of Breast Surgery (Surgical Oncology)

WenjieXia ( $\sim 741558330 @ q q . c o m$ )

Zhejiang Provincial People's Hospital

\section{Research Article}

Keywords: Pannexin 1 (PANX1), Neutrophils, Adenosine, Tumor microenvironment (TME), Triple-negative breast cancer (TNBC)

Posted Date: September 24th, 2021

DOI: https://doi.org/10.21203/rs.3.rs-899442/v1

License: (c) (i) This work is licensed under a Creative Commons Attribution 4.0 International License. Read Full License 


\section{Abstract}

High adenosine levels are an important characteristic of the tumor microenvironment (TME) in triplenegative breast cancer (TNBC). Pannexin 1 (PANX1) can release intracellular ATP to the extracellular space and elevate extracellular ATP (exATP) levels under physiological conditions. We performed public database bioinformatics analysis, surgical specimen histological validation, RNA sequencing, and exATP/extracellular adenosine (exADO) assays in vitro and in vivo to reveal the role of PANX1 in regulating the immune microenvironment of TNBC. Our results revealed that PANX1 was highly expressed and acted as a poor prognostic factor in TNBC. The PANX1 expression level was positively correlated with exATP and exADO levels in the TNBC TME. PANX1 promoted infiltration of tumor-associated neutrophils (TANs) through exATP, and TANs highly expressed ENTPD1 (CD39) / NT5E (CD73). This study suggests that high PANX1 expression could promote TAN accumulation and adenosine production in the TNBC TME which have been shown to induce local immunosuppression.

\section{Introduction}

Breast cancer is the most common malignant tumor in women, and triple-negative breast cancer (TNBC) molecular subtype, which accounts for $15-20 \%$ of all breast cancers, is characterized by high invasiveness and ease of recurrence and metastasis and is associated with a poor prognosis due to lack of specific treatments (1). Compared with other subtypes, TNBC is characterized by abundant immune cell infiltration (2), and the immune status of the tumor microenvironment (TME) has a significant impact on the treatment and prognosis of $\operatorname{TNBC}(3,4)$. Therefore, it is of clinical importance to investigate the key regulatory genes related to the immune microenvironment of TNBC.

Pannexin 1 (PANX1), a member of the gap junction protein family, forms hemichannels in cell membranes as hexamers or gap junction channels between cells, which mediates the release of intracellular ATP to the extracellular microenvironment $(5,6)$. Extracellular ATP (exATP) and its metabolite extracellular adenosine (exADO) are important factors that regulate the local immune TME (7). Under physiological conditions, exATP released by PANX1 promotes innate and adaptive immune responses by attracting immune cells (8). In the TME, this process is disrupted, and exATP is rapidly metabolized by the nucleotidases ENTPD1 (CD39) and NT5E (CD73) to generate exADO (9). In the TME, exADO is a key factor that contributes to local immunosuppression (10). Recently, PANX1 expression was found to be important in suppressing airway inflammation in the asthma mouse model, and knockdown of PANX1 resulted in increased airway inflammation (11). In breast cancer, the way in which PANX1 affects tumorinfiltrating immune cells (TIICs) and the immune microenvironment by regulating exATP levels has not been reported.

This study revealed that TNBC tissues had high PANX1 expression, which promoted the recruitment of tumor-associated neutrophils (TANs) and the accumulation of exADO, forming an immunosuppressive TME. 


\section{Materials And Methods \\ Data acquisition}

The TCGA-BRCA patient cohort data were downloaded from the TCGA website (https://portal.gdc.cancer.gov/; https://www.cancer.gov/tcga), and the METABRIC (12) cohort data were downloaded from the cBioPortal website (https://www.cbioportal.org). The METABRIC and TCGA-BRCA samples were classified into molecular subtypes using the PAM50 algorithm (13). Single-cell TNBC RNAseq data (14) (GSE118389) were downloaded from the Gene Expression Omnibus (GEO) database (https://www.ncbi.nlm.nih.gov/geo/).

\section{Clinical specimen collection}

Formalin-fixed paraffin-embedded (FFPE) sections from 12 TNBC patients who underwent surgery at the Second Affiliated Hospital of Zhejiang University School of Medicine (SAHZU) were collected from March 2020 to December 2020. Fresh surgical tumor specimens with paired peripheral blood samples from 15 patients with TNBC and 12 patients with Luminal A cancer who underwent surgery at SAHZU were collected from March 2020 to July 2021. Related basic clinicopathological and survival information was also collected after receipt of informed consent and approval from the ethics committee.

\section{Cell lines and culture conditions}

The MDA-MB-231, HCC-1937, and MCF-7 human breast cancer cell lines were all obtained from the American Type Culture Collection (ATCC, Manassas, VA, USA) and maintained under standard conditions according to ATCC.

\section{Bulk transcription data analysis}

For TCGA-BRCA and METABRIC data, the relative proportions of tumor-infiltrating immune cells were inferred using TIMER $(15,16)$ (V1: https://cistrome.shinyapps.io/timer, V2: http://timer.compgenomics.org/) and CIBERSORT LM22 (17) (22 immune cell reference profiles:

https://cibersort.stanford.edu/). For RNA-seq data of fresh surgical specimens, the fractions of tumorinfiltrating immune cells were inferred using TIMER V2 (http://timer.comp-genomics.org/) and quanTIseq (18). GEPIA2 (19) (https://gepia2.cancer-pku.cn/) was used to detect the top 250 genes related to PANX1. A list of human immune-related genes was derived through the Immunology Database and Analysis Portal (ImmPort) (20) (https://www.immport.org/). The survival analysis (overall survival, OS) was performed using the web server tool KM plotter (21) (https://kmplot.com/analysis/). All packages used in this study were run in R environment 4.0.5. Gene Ontology analyses were performed using the clusterProfiler R package (22).

\section{Single cell transcription data analysis}

The TNBC single-cell dataset (23) was downloaded from the Broad Institute Single Cell Portal (https://singlecell.broadinstitute.org/single_cell/study/SCP1106/). The dataset was analyzed using the 
Seurat R package. Nonlinear dimensional reduction (tSNE) was used to visualize clustering results. Epithelial cell clusters with highly variable CNVs were determined to be malignant by the inferCNV R package. The SingleR R package was used for cell type annotation, and the default annotated file (Wu_EMBO_metadata.csv) was used as a reference. The single-cell dataset was divided into PANX1 high and low expression groups according to the relative PANX1 expression level of each tumor sample in the count matrix (PANX1 low expression group: CID4513, CID4515 and CID44971; PANX1 high expression group: CID44041 and CID44991).

\section{Immunofluorescence staining}

Gene colocalization was validated by monoclonal antibody-based immunofluorescence. FFPE sections were subjected to antigen retrieval by heating the slides in citrate buffer for 2 minutes, after which they were incubated with primary antibodies (anti-human PANX1 and MPO antibodies) at $4^{\circ} \mathrm{C}$ overnight. Then, the slides were incubated with fluorescein-labeled secondary antibodies at room temperature, stained with DAPI and photographed under a laser confocal microscope (OLYMPUS IX83-FV3000-OSR).

\section{Immunohistochemical staining}

Gene expression was validated by monoclonal antibody-based immunohistochemistry. Immunohistochemical staining of FFPE slides, which were deparaffinized and rehydrated before the antigen retrieval step, was performed using the Envision method. Endogenous peroxidase was blocked by incubating the slides with $3 \% \mathrm{H}_{2} \mathrm{O}_{2}$. FFPE slides were heated in citrate buffer for 2 minutes, incubated with primary antibodies (anti-human PANX1/ENTPD1/NT5E antibodies) at $4^{\circ} \mathrm{C}$ overnight, and then incubated with the secondary antibody for 30 minutes at room temperature. 3,3'-Diaminobenzidine (DAB) chromogen (Zhongshan Jinqiao Biotech, Beijing, China) was used for visualization. The intensity and frequency were used as evaluation indexes based on PANX1 staining. The expression intensity was divided into 4 subgroups: negative (0), weakly positive (1), positive (2), and strong positive (3). The expression frequency was divided into 5 subgroups: $0 \%-10 \%$ (1), $11 \%-30 \%$ (2), $31 \%-50 \%$ (3), $51 \%-$ $75 \%$ (4), and 76\% - 100\% (5). PANX1 comprehensive score = intensity*frequency. The samples with the upper $50 \%$ of PANX1 comprehensive scores were defined as the PANX-high group, while the samples with the lower $50 \%$ of PANX1 comprehensive scores were defined as the PANX-low group.

\section{Neutrophil isolation}

Fresh surgical specimens were cut into small pieces and digested in medium containing $1 \mathrm{mg} / \mathrm{mL}$ collagenase IV (\#V900893, Sigma-Aldrich) at $37^{\circ} \mathrm{C}$ in a constant temperature shaker for $2 \mathrm{~h}$. The cell

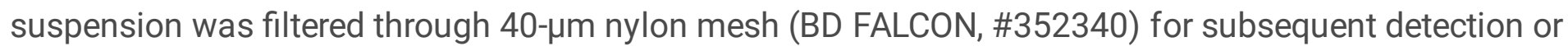
culture. The separation of neutrophils from surgical specimens and peripheral blood was performed with a human neutrophil isolation kit according to the manufacturer's protocol (Miltenyi Biotec, Auburn, CA, USA).

\section{RNA sequencing}


Human genomic DNA was isolated using a QIAamp DNA Mini Kit according to the manufacturer's protocol (Qiagen, Valencia, CA, USA). Single-end libraries were subsequently constructed using the standard protocol provided by BGI (BGI-Shenzhen) and were then sequenced on the BGISEQ-500 platform. Key immune-related gene expression was analyzed, and the heatmap was generated by the ComplexHeatmap R package.

\section{shRNA knockdown of PANX1}

MDA-MB-231 and HCC-1937 cells were transfected using the PANX1 human shRNA plasmid kit (OriGene, Rockville, MD, USA) (sequence: $5^{\prime}$ -

CGCAATGCTACTCCTGACAAACCTTGGCATGTCAAGAGCATGCCAAGGTTTGTCAGGAGTAGCATTGTT-3'). PANX1 expression was evaluated in control shRNA-transfected cells and was compared with that in nontransfected cells. Single-cell colonies of PANX shRNA-expressing cells were selected with puromycin and examined for PANX1 knockdown. Stable knockdown samples showed a 70-90\% reduction in PANX1 expression. Cells were maintained under puromycin selection pressure and were periodically examined for effective PANX1 knockdown using western blot.

\section{Extracellular adenosine assay}

In 24-well plates, $2 \times 10^{4}$ cells per well were allowed to adhere overnight after which they were removed by centrifugation at $4^{\circ} \mathrm{C}$ for 10 minutes; the supernatant was then collected for ATP/ADO detection assays. Fresh surgical TNBC tissues and normal specimens were cut into small pieces and digested in medium containing $1 \mathrm{mg} / \mathrm{mL}$ collagenase IV (\#V900893, Sigma-Aldrich) at $37^{\circ} \mathrm{C}$ at a constant temperature. The cell suspension was then filtered through 40- $\mu$ m nylon mesh (\#352340, BD FALCON) for subsequent ATP/ADO detection assays. ATP/ADO levels were measured using the ATP/ADO Assay Kit (fluorometric) (Abcam, Cambridge, MA, USA) according to the manufacturer's protocol.

\section{Statistical analyses}

Statistical significance was determined using unpaired two-tailed Student's t-tests and analysis of variance (ANOVA) followed by Tukey's test. For all in vitro assays, data are representative of at least three independent experiments, which each included three technical replicates unless otherwise stated. Differences in PANX1 expression in different subtypes were assessed using one-way ANOVA (Tukey's test). Paired differences in ATP/ADO levels and ENTPD1/NT5E expression between different groups were assessed using two-tailed t-tests. A log-rank test was performed using the online tool KM plotter to assess PANX1 as a survival biomarker. Statistical analyses were performed using GraphPad Prism software (version 9.0) and R software (version 4.0.5, R Core Team, http://www.R-project.org/). The results are given as the mean \pm S.D. and $p$ values $<0.05$ were considered significant (unless otherwise stated). The details of the statistical analysis are provided in the results section and figure legends.

\section{Results}




\section{PANX1 was highly expressed in TNBC and was negatively correlated with TNBC prognosis}

Compared with normal tissues, we found that PANX1 was highly expressed in breast cancer (BRCA), colon adenocarcinoma (COAD), esophageal carcinoma (ESCA), head and neck squamous cell carcinoma (HNSC), kidney chromophobe (KICH), lung adenocarcinoma (LUAD), lung squamous cell carcinoma (LUSC), and stomach adenocarcinoma (STAD) based on the web tool TIMER (TCGA-BRCA data) ( $p<$ 0.001 was significant) (Fig. 1A). To further investigate the role of PANX1 expression in breast cancer, we analyzed TCGA-BRCA and METABRIC transcriptome data and found that PANX1 was highly expressed in the basal-like subtype (PAM50 model) (Fig. 1B-C). The prognostic value of PANX1 in different breast cancer molecular types was analyzed using the KM plotter web tool, and high PANX1 expression suggested poor prognosis in terms of overall survival in TNBC $(p=0.015)$ (Fig. 1D). In the other three molecular subtypes (Luminal A \& B and HER2-enriched), no significant correlation was observed between PANX1 expression and overall survival $(p>0.05)$ (Fig. 1D). The above results suggested that PANX1 was highly expressed and was associated with a poor prognosis in TNBC.

\section{PANX1 promoted high ENTPD1/NT5E expression in the TME}

PANX1 is acknowledged as a dominant regulator of exATP release, while exATP and its metabolite exADO are believed to induce an immune-suppressive TME (9). Analysis of TCGA-BRCA basal-like subtype transcriptome data revealed that the PANX1 high expression (top 50\%) group had higher ENTPD1 and NT5E expression levels than the PANX1 low expression (bottom 50\%) group $(p<0.001)$ (Fig. 2A). Further linear analysis suggested a positive correlation between PANX1 expression and ENTPD1 and NT5E expression in TCGA-BRCA basal-like subtype transcriptome data $(p<0.001)$ (Fig. 2B). The RNA-seq data of surgical breast cancer specimens (TNBC PANX1 high: $n=3$; TNBC PANX1 low: $n=3$; Luminal subtype: $\mathrm{n}=3$ ) suggested higher expression of ENTPD1 and NT5E in the TNBC PANX1-high group compared to TNBC PANX1-low and Luminal group $(p<0.05)$ (Fig. 2C). According to immunohistochemistry of TNBC surgical specimens, the expressions of ENTPD1 and NT5E were higher in the PANX1 high group than in the PANX1 low group (Fig. 2D). The above results indicated that high PANX1 expression promoted high ENTPD1/NT5E expression in the TNBC TME.

\section{PANX1 expression was positively correlated with TAN infiltration in TNBC}

Using CIBERSORT LM22, we analyzed the effect of PANX1 expression in the TCGA-BRCA basal-like subtype on the tumor immune microenvironment. We found that the abundances of infiltrating neutrophils $(p<0.05)$, resting memory $\mathrm{CD}^{+} \mathrm{T}$ cells $(p<0.05)$, follicular helper T cells $(p<0.05)$, monocytes $(p<0.05), \mathrm{CD} 8^{+}$T cells $(p<0.05)$, and activated natural killer (NK) cells $(p<0.05)$ were significantly different between tumors with high PANX1 expression (top 50\%) and those with low PANX1 
expression (bottom 50\%) (Fig. 3A). The top 250 coexpressed PANX1-related genes were obtained through GEPIA2, and the gene coexpression network was constructed by GeneMANIA after intersection with the ImmPort gene set (Fig. 3B). Furthermore, gene ontology (GO) analysis suggested that PANX1 and its coexpressed genes were related to granulocyte chemotaxis (Fig. 3C). The relationship between PANX1 expression and TIICs was assessed using TIMER in the TCGA-BRCA basal-like subtype data, and a positive correlation was found between PANX1 and the infiltration level of TANs $(p<0.05)$ (Fig. 3D). The above results suggest a positive correlation between PANX1 expression and neutrophil infiltration in TNBC. The coexpression of PANX1 and myeloperoxidase (MPO) in TNBC paraffin-embedded surgical specimens was observed using immunofluorescence. PANX1 and MPO expression was positive correlated, indicating that high PANX1 expression promoted TAN infiltration in the TNBC TME (Fig. 3E). However, the way in which PANX1 establishes an immunosuppressive microenvironment with TANs should be further explored.

\section{Immunosuppressive TANs demonstrated more infiltration in TNBC with high PANX1 expression}

The quanTIseq and TIMER deconvolution methods have high deconvolution performance for RNA-seq data from different tumor types and can be suitable tools for further exploration of tumor-infiltrating neutrophils (24). RNA-seq data from fresh surgical specimens were converted into infiltrating immune cell information by quanTIseq and TIMER. The results revealed that TNBC with high PANX1 expression had more infiltrating TANs than TNBC with low PANX1 expression and the Luminal subtype $(p<0.05$ for TIMER; $p=0.106$ for quanTIseq) (Fig. 4A-C). The relationship between ENTPD1/NT5E expression and the infiltration level of TANs in the basal-like subtype was evaluated by TIMER, and ENTPD1/NT5E expression was positively correlated with the infiltration level of TANs $(p<0.01)$ (Fig. 4D). Neutrophils were purified from fresh TNBC surgical specimens and paired peripheral blood samples, and the transcriptome analysis of TANs revealed higher expression of the nucleotidases ENTPD1 and NT5E ( $p<$ $0.05)$ and high expression of the immunosuppressive cell recruitment-related cytokines CCL2 and CCL17 $(p<0.05)$ compared with the peripheral blood (Fig. 4E). The above results suggest that PANX1 promotes the recruitment of TANs in TME, and TANs have the ability to convert exATP to exADO with highly expressing ENTPD1 and NT5E.

\section{High PANX1 expression induced a high exADO immunosuppressive TME in TNBC}

To clarify whether high PANX1 expression in TNBC could establish an immunosuppressive TME with local high exADO levels, we measured the exATP and exADO levels in the supernatant of cultured breast cancer cell lines and found that exATP and exADO levels in the MDA-MB-231 and HCC-1937 (basal-like subtype cell lines) cell culture media were significantly higher than those in the MCF-7 (Luminal subtype cell line) cell culture media $(p<0.05)$ (Fig. 5A). PANX1 knock down in the MDA-MB-231 and HCC-1937 cell lines led to a downregulation of the exATP and exADO levels in the cell culture media $(p<0.01)$ (Fig. 5A). 
The exATP and exADO levels in the digested tissue supernatant of TNBC surgical samples were significantly higher than those in Luminal A breast cancer surgical samples $(n=9)(p<0.05)$ (Fig. 5B). The correlation between PANX1 expression and TIIC infiltration in the TCGA-BRCA basal-like subtype was analyzed by TIMER. The results indicated that PANX1 expression was positively correlated with the infiltration level of regulatory T cells (Tregs), M2-like macrophages and myeloid-derived suppressor cells (MDSCs) $(p<0.05)$ and was negatively correlated with the infiltration level of $\mathrm{CD}^{+} \mathrm{T}$ cells and NK cells ( $p$ $<0.05$ ) (Fig. 4C). The TNBC single-cell transcriptome data were analyzed (23) and it was found that tumor samples with high PANX1 expression had lower infiltration of B cells, $\mathrm{CD} 4^{+} \mathrm{T}$ cells, $\mathrm{CD} 8^{+} \mathrm{T}$ cells, myeloid cells, NK cells and NK T cells and higher infiltration of cancer-associated fibroblasts (CAFs), plasma cells and Tregs (Fig. 4D-E). The above results suggested that PANX1 is a key gene responsible for exADO accumulation and establishment of an immunosuppressive TME in TNBC.

\section{Discussion}

This study suggests that PANX1 is highly expressed in TNBC and is a prognostic factor associated with poor prognosis in TNBC. PANX1 promotes TAN infiltration by increasing exATP levels in TNBC, and TANs highly express ENTPD1/NT5E, which synergistically contributes to an immunosuppressive environment with high exADO levels and promotes TNBC progression.

Malignant tumors are known as "never healing wounds", and chronic inflammation is one of the key features of malignancy. Chronic inflammatory processes are involved in tumorigenesis and tumor progression. Purine nucleosides (ATP and ADO) exert a strong immunomodulatory ability in the TME, and exATP/exADO can regulate local immune responses by activating immune cell purinergic $P 2$ receptors. Previous studies have focused on the role of the nucleotidases ENTPD1 and NT5E in exADO production and the establishment of an immunosuppressive microenvironment $(25,26)$. However, the source and regulatory mechanism of exATP in the TME, which is the substrate for exADO, has not been as well studied.

Stewart et al. found that PANX1 expression was required for breast development during lactation and that high PANX1 expression was associated with worse clinical outcomes in breast cancer (27). In other malignant tumors, PANX1 exhibited procarcinogenic effects in hepatocellular carcinoma (28), testicular carcinoma (29) and melanoma (30) and anticarcinogenic effects in glioma (31) and rhabdomyosarcoma (32). The above results suggest that the role of PANX1 in tumorigenesis might be complex and might depend on the tumor type and stage. In this study, we investigated the effect of PANX1 in TNBC primary lesions. We found that PANX1 was highly expressed in TNBC and could increase the exATP level in the TME and that high PANX1 expression was associated with poor prognosis in TNBC. These prognosis results are consistent with those in previous breast cancer studies $(29,33)$. However, previous studies have primarily focused on the effects of PANX1 on breast cancer cells (33), and the role of PANX1 in the formation of the immunosuppressive microenvironment has not been fully explored. In vitro studies had shown that PANX1 promoted the activation of NLRP3 (NOD-, LRR- and pyrin domain-containing protein 3) inflammasome and increased the level of interleukin $1 \beta$ (IL-1 $\beta$ ) in the local microenvironment (34). 
In this study, we found that the abundance of infiltrating neutrophils was significantly higher in the PANX1 high expression TNBC and that the genes coexpressed with PANX1 were related to granulocyte chemotaxis. In the early stage of tumor development, as the first immune cells to enter the tumor microenvironment, neutrophils mediate subsequent immune responses and regulatory processes $(35,36)$. The exATP secreted by PANX1 is considered to be an important damage-associated molecular pattern (DAMP) signal (37) that could lead to neutrophil recruitment (8). exATP could exacerbate the local immune response by mediating NLRP3 inflammasome activation and IL-1 $\beta$ secretion via the P2Y7 receptor (P2X7R) on neutrophils (38). exATP could also delay neutrophil apoptosis via the P2Y11 receptor (P2Y11R) (39). This study also demonstrated that TANs expressing high levels of ENTPD1/NT5E could promote the hydrolysis of exATP to exADO to aggravate the immunosuppressive TME. This is consistent with the results of studies of inflammatory states $(40,41)$. Chen et al. demonstrated that neutrophils were chemotactic to exATP and hydrolyzed exATP to exADO by NT5E to promote cell migration $(41,42)$. In addition, exADO inhibits neutrophil adhesion and the release of TNF- $a$ and chemokines from LPSstimulated neutrophils (43). Neutrophil-expressed adenosine A2A receptor (A2AR) could inhibit the neutrophil recruitment cascade (44). Previous studies also revealed that the purinergic receptor P2Y6 receptor (P2Y6R) (45), adenosine A2B receptor (A2BR) (46), and adenosine A3 receptor (A3R) (47) on neutrophils are involved in the regulation of neutrophil extracellular traps (NETs) in inflammatory states. Whether exATP/exADO can regulate NETs or the N1/N2-like subtype transition of TANs through purinergic receptors and thus affect the development and metastasis of breast cancer requires further investigation.

In this study, we demonstrated that ENTPD1 and NT5E expression was higher in the PANX1 high expression TNBC and PANX1 upregulated exADO levels in the TME. PANX1 is an important immunomodulator in the TME. When the results of single-cell transcriptome data analysis were combined with TCGA-BRCA data analysis in TNBC, we found that PANX1 expression was negatively correlated with the infiltration levels of $\mathrm{CD} 8^{+} \mathrm{T}$ cells and NK cells and was positively correlated with the infiltration levels of Tregs. The levels of CAFs were also higher in PANX1 high expression TNBC. A similar phenomenon has been observed during renal injury. Higher PANX1 expression caused an increase in exATP, which was further catabolized by ENTPD1 and then converted to adenosine by NT5E (48). PANX1 plays an important role in the maintenance of chronic inflammation. Targeting PANX1 could inhibit tumorassociated inflammatory responses and reverse the local immunosuppressive microenvironment. Therefore, in-depth investigations of PANX1 may help elucidate tumor-inflammation interactions.

In summary, the high expression of PANX1 in TNBC was a poor prognostic factor and positively correlated with the exADO level. PANX1 could promote TAN infiltration through exATP secretion, and the high expression of ENTPD1/NT5E in TANs could synergistically establish an immunosuppressive TME with high exADO levels. In this study, the relationship between high exATP/exADO levels and TANs was investigated to elucidate the properties of PANX1 and its ability to reshape the metabolicimmunosuppressive TME and provide new targets and strategies for TNBC treatment.

\section{Abbreviations}




\begin{tabular}{ll} 
A2AR & Adenosine A2A receptor \\
\hline A2BR & Adenosine A2B receptor \\
\hline A3R & Adenosine A3 receptor \\
\hline BRCA & Breast cancer \\
\hline CAF & Cancer-associated fibroblast \\
\hline COAD & Colon adenocarcinoma \\
\hline DAMP & Damage-associated molecular pattern \\
\hline ECM & Extracellular matrix \\
\hline EMT & Epithelial-mesenchymal transition \\
\hline ESCA & Esophageal carcinoma \\
\hline exADO & Extracellular adenosine \\
\hline exATP & Extracellular ATP \\
\hline FFPE & Formalin-fixed paraffin-embedded \\
\hline HNSC & Head and neck squamous cell carcinoma \\
\hline IL & Interleukin \\
\hline KICH & Kidney chromophobe \\
\hline LUAD & Lung adenocarcinoma \\
\hline LUSC & Lung squamous cell carcinoma \\
\hline MDSC & Myeloid-derived suppressor cell \\
\hline MPO & Myeloperoxidase \\
\hline NETs & Neutrophil extracellular traps \\
\hline NK & Natural killer \\
\hline NLRP3 & NOD-, LRR- and pyrin domain-containing protein 3 \\
\hline P2Y11R & P2Y11 receptor \\
\hline P2Y6R & P2Y6 receptor \\
\hline P2Y7R & P2Y7 receptor \\
\hline PANX1 & Pannexin 1 \\
\hline PTAD & Peripheral blood neutrophil \\
\hline Stomach adenocarcinoma \\
\hline PBN
\end{tabular}




\begin{tabular}{ll} 
TAN & Tumor-associated neutrophil \\
\hline TIIC & Tumor-infiltrating immune cell \\
\hline TME & Tumor microenvironment \\
\hline TNBC & Triple-negative breast cancer \\
\hline Treg & Regulatory T cell \\
\hline
\end{tabular}

\section{Declarations}

\section{Ethics Approval and Consent to Participate}

Ethical approval for this study was received from the Ethical Committee of Second Affiliated Hospital, Zhejiang University School of Medicine (No. SHENYAN 2020-337, approved March 2020) (Scanned original file has been uploaded Zenodo: https://doi.org/10.5281/zenodo.5168915).

Written informed consent was obtained from all clinical sample donors according to the guidelines approved by the Ethical Committee of the Second Affiliated Hospital, Zhejiang University School of Medicine and all methods were carried out in accordance with relevant guidelines and regulations.

\section{Consent for publication}

Not applicable.

\section{Availability of Data and Materials}

The data from previously reported studies and datasets, which supporting this research, have been cited in the manuscript. All the raw data and results generated during and/or analyzed during the current study have been publicly deposited in the Zenodo repository and can be accessed at https://doi.org/10.5281/zenodo.5168915.

\section{Competing Interest}

The authors declare no conflicts of interest.

\section{Funding}

This work was supported by the Zhejiang Medical and Health Science and Technology Plan Project (No: 2019RC040), the Natural Science Foundation of Zhejiang Province (No: LQ20H160064) and the Fundamental Research Funds for the Central Universities (No: 2021FZ203-02-08). 


\section{Authors' Contributions}

WZ designed the study. WJ, BZ and FJ coordinated data acquisition, performed the bioinformatics analysis, wrote the first draft of the manuscript, and revised the manuscript. JX and $\mathrm{HH}$ completed experiments on cell lines and tissue samples related to this project. WJ and $\mathrm{CN}$ were involved in the study design and critically revised the manuscript. All authors read and approved the final manuscript.

\section{Acknowledgments}

The authors thank Dr. Jian Huang, Dr. Zhigang Chen, Dr. Pu Chen, Dr. Ke Wang, and Dr. Zhigang Zhang for their helpful advice.

\section{References}

1. Abramson VG, Lehmann BD, Ballinger TJ, Pietenpol JA. Subtyping of triple-negative breast cancer: implications for therapy. Cancer. 2015;121(1):8-16.

2. Bareche $Y$, Venet $D$, Ignatiadis $M$, Aftimos $P$, Piccart $M$, Rothe $F$, et al. Unravelling triple-negative breast cancer molecular heterogeneity using an integrative multiomic analysis. Ann Oncol. 2018;29(4):895902.

3. Gao G, Wang Z, Qu X, Zhang Z. Prognostic value of tumor-infiltrating lymphocytes in patients with triple-negative breast cancer: a systematic review and meta-analysis. BMC Cancer. 2020;20(1):179.

4. Perez EA, Ballman KV, Tenner KS, Thompson EA, Badve SS, Bailey H, et al. Association of Stromal Tumor-Infiltrating Lymphocytes With Recurrence-Free Survival in the N9831 Adjuvant Trial in Patients With Early-Stage HER2-Positive Breast Cancer. JAMA Oncol. 2016;2(1):56-64.

5. Chekeni FB, Elliott MR, Sandilos JK, Walk SF, Kinchen JM, Lazarowski ER, et al. Pannexin 1 channels mediate 'find-me' signal release and membrane permeability during apoptosis. Nature. 2010;467(7317):863-7.

6. Lohman AW, Leskov IL, Butcher JT, Johnstone SR, Stokes TA, Begandt D, et al. Pannexin 1 channels regulate leukocyte emigration through the venous endothelium during acute inflammation. Nat Commun. 2015;6:7965.

7. Jacob F, Perez Novo C, Bachert C, Van Crombruggen K. Purinergic signaling in inflammatory cells: P2 receptor expression, functional effects, and modulation of inflammatory responses. Purinergic signalling. 2013;9(3):285-306.

8. Yang D, He Y, Muñoz-Planillo R, Liu Q, Núñez G. Caspase-11 Requires the Pannexin-1 Channel and the Purinergic P2X7 Pore to Mediate Pyroptosis and Endotoxic Shock. Immunity. 2015;43(5):923-32.

9. Vijayan D, Young A, Teng MWL, Smyth MJ. Targeting immunosuppressive adenosine in cancer. Nat Rev Cancer. 2017;17(12):709-24. 
10. Feng LL, Cai YQ, Zhu MC, Xing LJ, Wang X. The yin and yang functions of extracellular ATP and adenosine in tumor immunity. Cancer Cell Int. 2020;20:110.

11. Medina CB, Chiu YH, Stremska ME, Lucas CD, Poon I, Tung KS, et al. Pannexin 1 channels facilitate communication between $T$ cells to restrict the severity of airway inflammation. Immunity. 2021;54(8):1715-27.e7.

12. Curtis C, Shah SP, Chin SF, Turashvili G, Rueda OM, Dunning MJ, et al. The genomic and transcriptomic architecture of 2,000 breast tumours reveals novel subgroups. Nature. 2012;486(7403):346-52.

13. Jørgensen CLT, Larsson AM, Forsare C, Aaltonen K, Jansson S, Bradshaw R, et al. PAM50 Intrinsic Subtype Profiles in Primary and Metastatic Breast Cancer Show a Significant Shift toward More Aggressive Subtypes with Prognostic Implications. Cancers (Basel). 2021;13(7).

14. Karaayvaz M, Cristea S, Gillespie SM, Patel AP, Mylvaganam R, Luo CC, et al. Unravelling subclonal heterogeneity and aggressive disease states in TNBC through single-cell RNA-sEq. Nat Commun. 2018;9(1):3588.

15. Li T, Fu J, Zeng Z, Cohen D, Li J, Chen Q, et al. TIMER2.0 for analysis of tumor-infiltrating immune cells. Nucleic Acids Res. 2020;48(W1):W509-w14.

16. Li T, Fan J, Wang B, Traugh N, Chen Q, Liu JS, et al. TIMER: A Web Server for Comprehensive Analysis of Tumor-Infiltrating Immune Cells. Cancer Res. 2017;77(21):e108-e10.

17. Newman AM, Liu CL, Green MR, Gentles AJ, Feng W, Xu Y, et al. Robust enumeration of cell subsets from tissue expression profiles. Nature methods. 2015;12(5):453-7.

18. Finotello F, Mayer C, Plattner C, Laschober G, Rieder D, Hackl H, et al. Molecular and pharmacological modulators of the tumor immune contexture revealed by deconvolution of RNA-seq data. Genome Med. 2019;11(1):34.

19. Tang Z, Kang B, Li C, Chen T, Zhang Z. GEPIA2: an enhanced web server for large-scale expression profiling and interactive analysis. Nucleic Acids Res. 2019;47(W1):W556-w60.

20. Bhattacharya S, Dunn P, Thomas CG, Smith B, Schaefer H, Chen J, et al. ImmPort, toward repurposing of open access immunological assay data for translational and clinical research. Sci Data. 2018;5:180015.

21. Nagy Á, Munkácsy G, Győrffy B. Pancancer survival analysis of cancer hallmark genes. Scientific reports. $2021 ; 11(1): 6047$.

22. Yu G, Wang LG, Han Y, He QY. clusterProfiler: an R package for comparing biological themes among gene clusters. Omics. 2012;16(5):284-7.

23. Wu SZ, Roden DL, Wang C, Holliday H, Harvey K, Cazet AS, et al. Stromal cell diversity associated with immune evasion in human triple-negative breast cancer. The EMBO journal. 2020;39(19):e104063.

24. Finotello F, Trajanoski Z. Quantifying tumor-infiltrating immune cells from transcriptomics data. Cancer Immunol Immunother. 2018;67(7):1031-40. 
25. Buisseret L, Pommey S, Allard B, Garaud S, Bergeron M, Cousineau I, et al. Clinical significance of CD73 in triple-negative breast cancer: multiplex analysis of a phase III clinical trial. Ann Oncol. 2018;29(4):1056-62.

26. Allard B, Allard D, Buisseret L, Stagg J. The adenosine pathway in immuno-oncology. Nat Rev Clin Oncol. 2020;17(10):611-29.

27. Stewart MK, Plante I, Penuela S, Laird DW. Loss of Panx1 Impairs Mammary Gland Development at Lactation: Implications for Breast Tumorigenesis. PloS one. 2016;11(4):e0154162.

28. Shi G, Liu C, Yang Y, Song L, Liu X, Wang C, et al. Panx1 promotes invasion-metastasis cascade in hepatocellular carcinoma. J Cancer. 2019;10(23):5681-8.

29. Liu H, Yuan M, Yao Y, Wu D, Dong S, Tong X. In vitro effect of Pannexin 1 channel on the invasion and migration of I-10 testicular cancer cells via ERK1/2 signaling pathway. Biomed Pharmacother. 2019;117:109090.

30. Penuela S, Gyenis L, Ablack A, Churko JM, Berger AC, Litchfield DW, et al. Loss of pannexin 1 attenuates melanoma progression by reversion to a melanocytic phenotype. J Biol Chem. 2012;287(34):29184-93.

31. Lai CP, Bechberger JF, Thompson RJ, MacVicar BA, Bruzzone R, Naus CC. Tumor-suppressive effects of pannexin 1 in C6 glioma cells. Cancer Res. 2007;67(4):1545-54.

32. Xiang X, Langlois S, St-Pierre ME, Blinder A, Charron P, Graber TE, et al. Identification of pannexin 1regulated genes, interactome, and pathways in rhabdomyosarcoma and its tumor inhibitory interaction with AHNAK. Oncogene. 2021;40(10):1868-83.

33. Jalaleddine N, El-Hajjar L, Dakik H, Shaito A, Saliba J, Safi R, et al. Pannexin1 Is Associated with Enhanced Epithelial-To-Mesenchymal Transition in Human Patient Breast Cancer Tissues and in Breast Cancer Cell Lines. Cancers. 2019;11(12):1967.

34. Laird DW, Penuela S. Pannexin biology and emerging linkages to cancer. Trends Cancer. 2021.

35. Eruslanov EB. Phenotype and function of tumor-associated neutrophils and their subsets in earlystage human lung cancer. Cancer Immunol Immunother. 2017;66(8):997-1006.

36. Shaul ME, Fridlender ZG. Cancer-related circulating and tumor-associated neutrophils - subtypes, sources and function. FEBS J. 2018;285(23):4316-42.

37. Di Virgilio F, Sarti AC, Coutinho-Silva R. Purinergic signaling, DAMPs, and inflammation. Am J Physiol Cell Physiol. 2020;318(5):C832-c5.

38. Karmakar M, Katsnelson MA, Dubyak GR, Pearlman E. Neutrophil P2X7 receptors mediate NLRP3 inflammasome-dependent IL-1 $\beta$ secretion in response to ATP. Nat Commun. 2016;7:10555.

39. Vaughan KR, Stokes L, Prince LR, Marriott HM, Meis S, Kassack MU, et al. Inhibition of neutrophil apoptosis by ATP is mediated by the P2Y11 receptor. J Immunol. 2007;179(12):8544-53.

40. Antonioli L, Pacher P, Vizi ES, Haskó G. CD39 and CD73 in immunity and inflammation. Trends Mol Med. 2013;19(6):355-67.

41. Wang X, Chen D. Purinergic Regulation of Neutrophil Function. Front Immunol. 2018;9:399. 
42. Chen $\mathrm{Y}$, Corriden R, Inoue $\mathrm{Y}$, Yip L, Hashiguchi N, Zinkernagel A, et al. ATP release guides neutrophil chemotaxis via P2Y2 and A3 receptors. Science. 2006;314(5806):1792-5.

43. Barletta KE, Ley K, Mehrad B. Regulation of neutrophil function by adenosine. Arterioscler Thromb Vasc Biol. 2012;32(4):856-64.

44. Yago T, Tsukamoto H, Liu Z, Wang Y, Thompson LF, McEver RP. Multi-Inhibitory Effects of A2A Adenosine Receptor Signaling on Neutrophil Adhesion Under Flow. J Immunol. 2015;195(8):3880-9.

45. Sil P, Hayes CP, Reaves BJ, Breen P, Quinn S, Sokolove J, et al. P2Y6 Receptor Antagonist MRS2578 Inhibits Neutrophil Activation and Aggregated Neutrophil Extracellular Trap Formation Induced by Gout-Associated Monosodium Urate Crystals. J Immunol. 2017;198(1):428-42.

46. Barletta KE, Cagnina RE, Burdick MD, Linden J, Mehrad B. Adenosine A(2B) receptor deficiency promotes host defenses against gram-negative bacterial pneumonia. Am J Respir Crit Care Med. 2012;186(10):1044-50.

47. Corriden R, Self T, Akong-Moore K, Nizet V, Kellam B, Briddon SJ, et al. Adenosine-A3 receptors in neutrophil microdomains promote the formation of bacteria-tethering cytonemes. EMBO Rep. 2013;14(8):726-32.

48. Poudel N, Okusa MD. Pannexins in Acute Kidney Injury. Nephron. 2019;143(3):158-61.

\section{Figures}


A

Figure 1

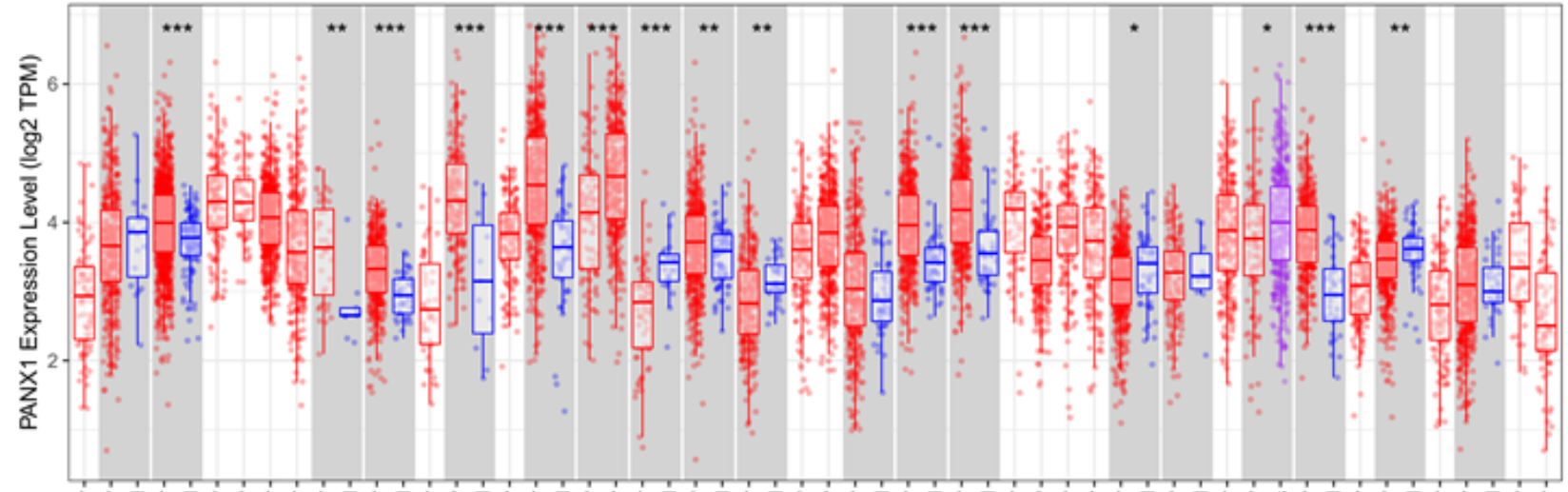

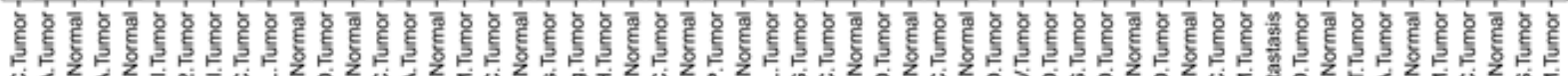

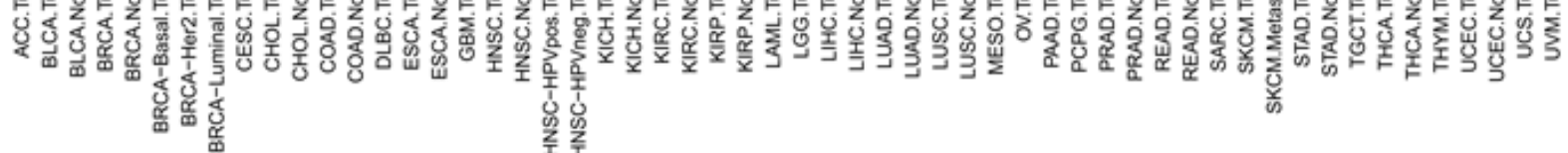

B

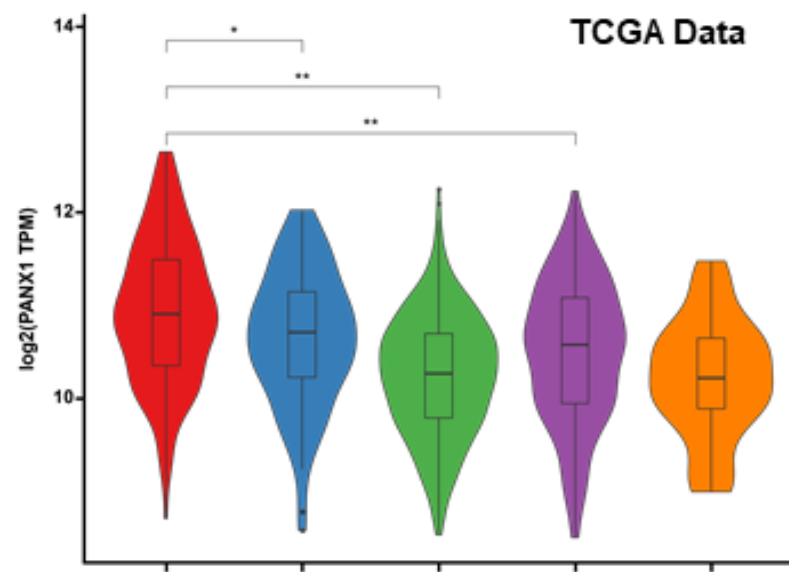

BRCA.Basal BRCA.Her2 BRCA.LUmA BRCA.LumB BRCA.Normal

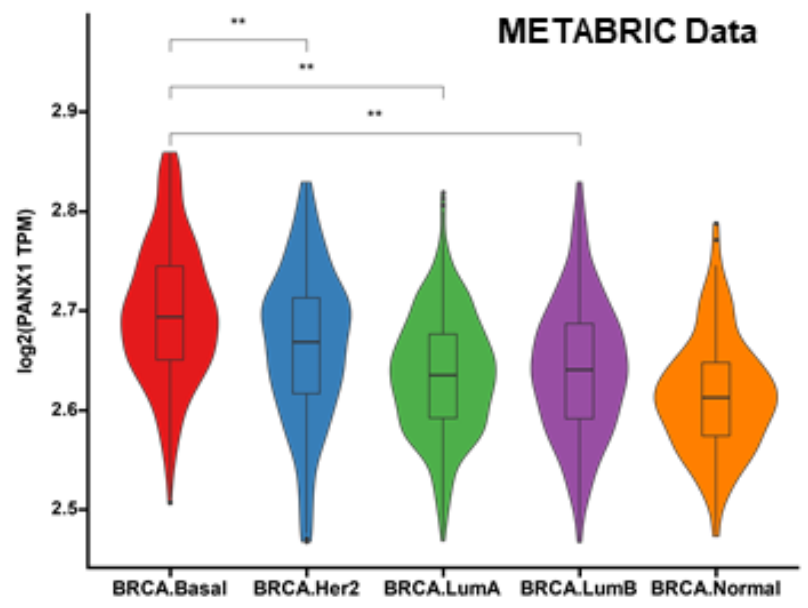

D
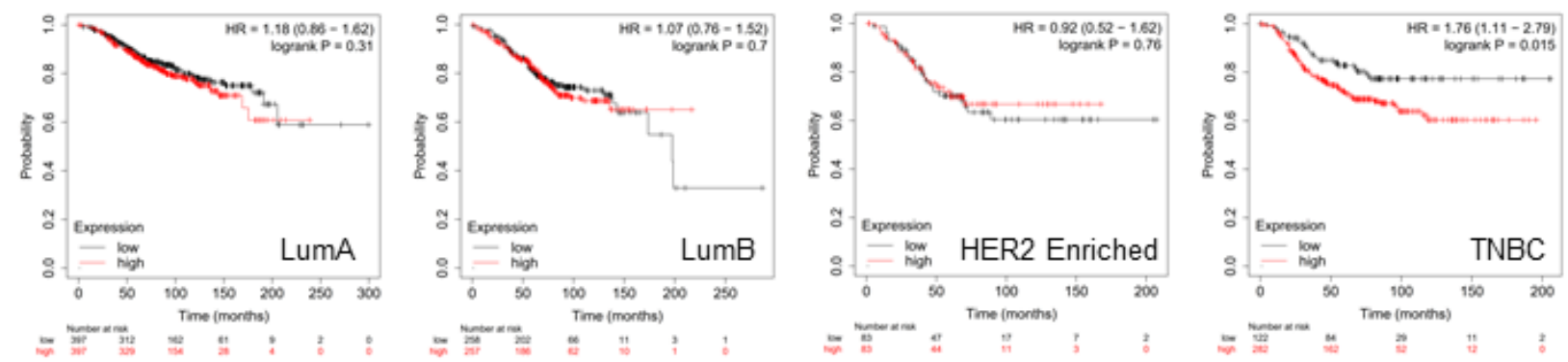

\section{Figure 1}

(A) PANX1 was highly expressed in breast cancer (BRCA), colon adenocarcinoma (COAD), esophageal carcinoma (ESCA), head and neck squamous cell carcinoma (HNSC), kidney chromophobe (KICH), lung adenocarcinoma (LUAD), lung squamous cell carcinoma (LUSC), and stomach adenocarcinoma (STAD) compared with normal tissues ( $p$ < 0.001 was significant) (TCGA-BRCA data; Red bar: tumor tissue; Blue bar: normal tissue); (B-C) PANX1 expression in different breast cancer molecular subtypes (TCGA-BRCA 
and METABRIC data, PAM50 algorithm); (D) Correlation between PANX1 expression and overall survival prognosis in different breast cancer molecular subtypes (Luminal A, Luminal B, HER2-enriched and TNBC). (TNBC, triple-negative breast cancer) (* $p<0.05 ; * \star p<0.01 ; * \star * p<0.001)$

A

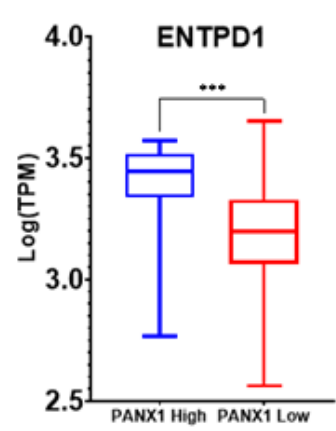

B
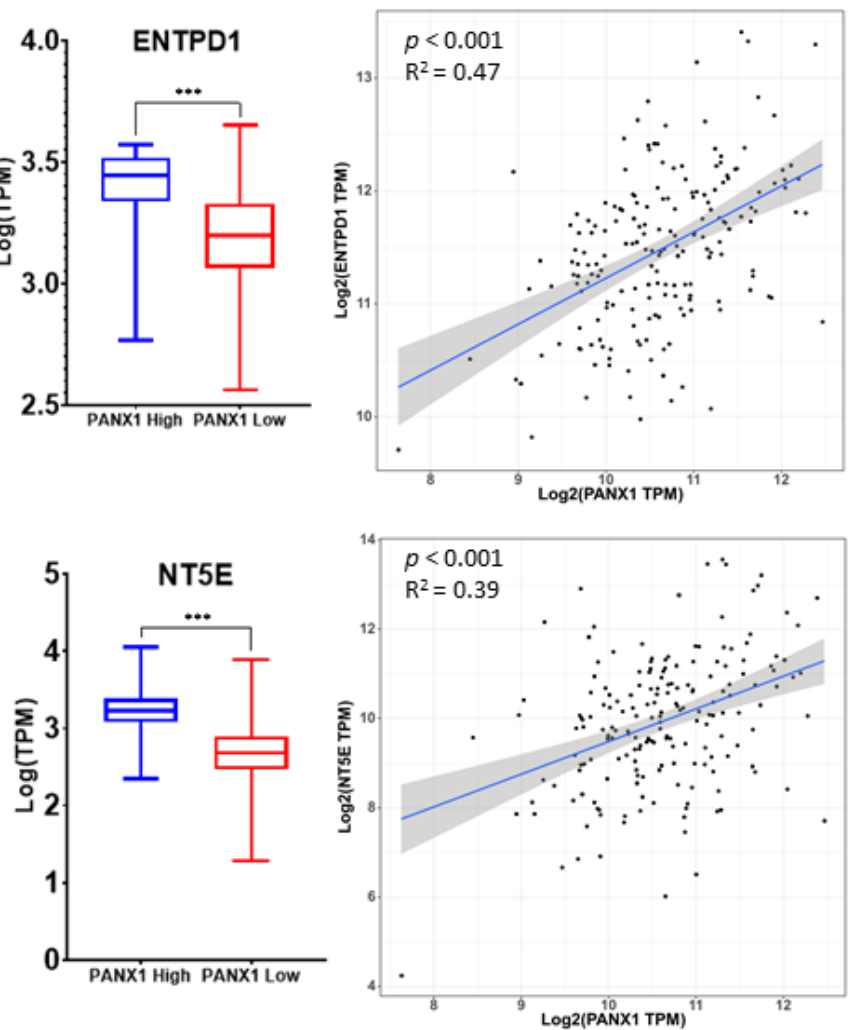

C

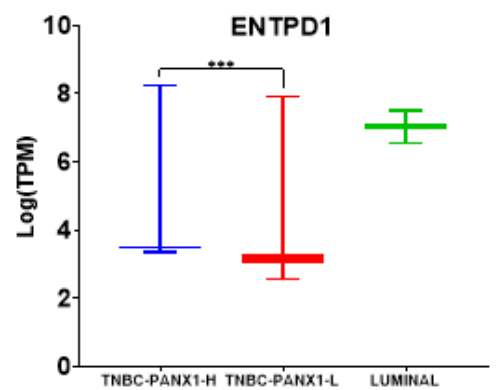

Figure 2

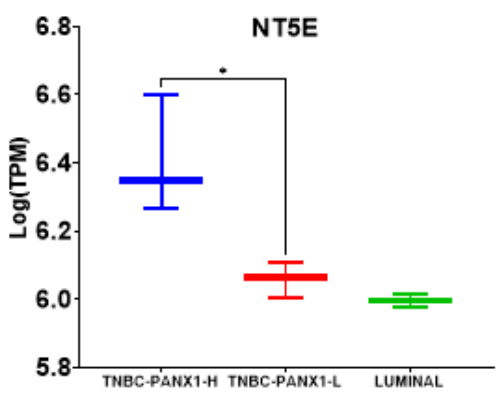

D

Figure 2

(A) ENTPD1 and NT5E expression in TNBC specimens with different levels of PANX1 expression $(\mathrm{p}<$ 0.01) (TCGA-BRCA basal-like subtype data); (B) ENTPD1 and NT5E expression was positively correlated with PANX1 expression in TNBC ( $p$ < 0.001) (TCGA-BRCA basal-like subtype data); (C) ENTPD1 and NT5E expression in breast cancer specimens (TNBC PANX1 high subgroup: $\mathrm{n}=3$; TNBC PANX1 low subgroup: $n=3$; Luminal subtype: $n=3)(p<0.05)$; (D) ENTPD1 and NT5E expression in TNBC surgical specimens with different PANX1 expression levels by immunohistochemistry at $10 \mathrm{X}$ and $20 \mathrm{X}$ magnifications. (TNBC, triple-negative breast cancer) (* $p<0.05 ; * \star \star p<0.001)$ 
A

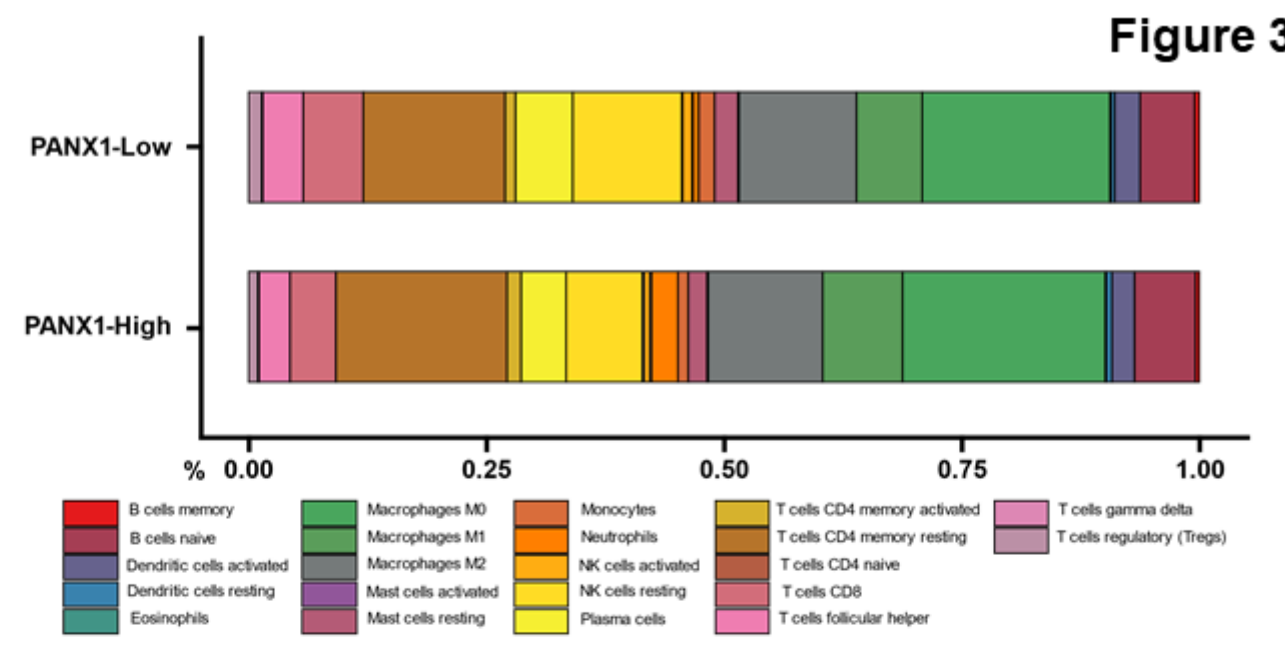

B

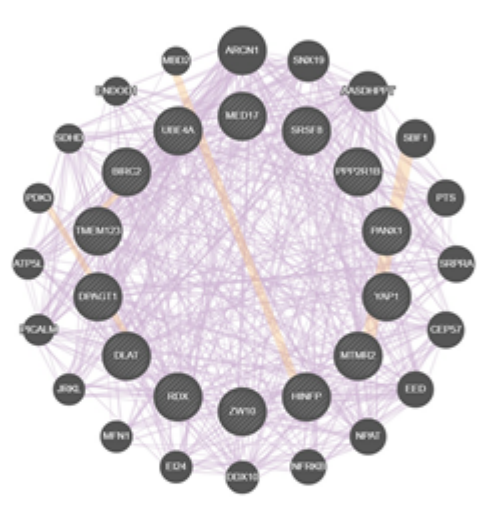

C

E
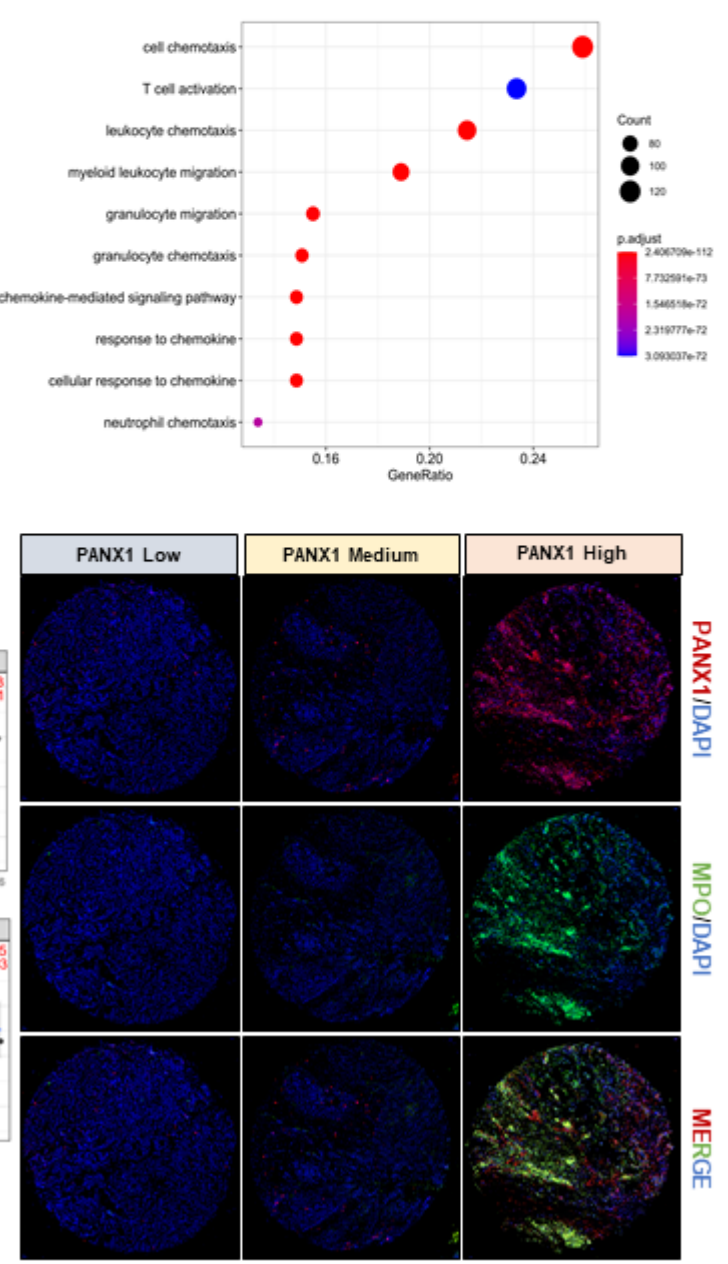

Figure 3

(A) CIBERSORT evaluated tumor-infiltrating immune cell (TIIC) differences under high (top 50\%)/low (bottom 50\%) PANX1 expression in TCGA-BRCA basal-like subtype; (B) PANX1 and its coexpression immune-related gene network; (C) GO analysis of PANX1 and its coexpressed immune-related genes; (D) TIMER analysis of PANX1 expression and TIIC correlation; (E) Immunofluorescence detection of 
PANX1/MPO (neutrophil marker) coexpression in TNBC paraffin-embedded pathological specimens. (DAPI, 4',6-diamidino-phenylindole; MPO, myeloperoxidase; TNBC, triple-negative breast cancer)

A $\cdot$
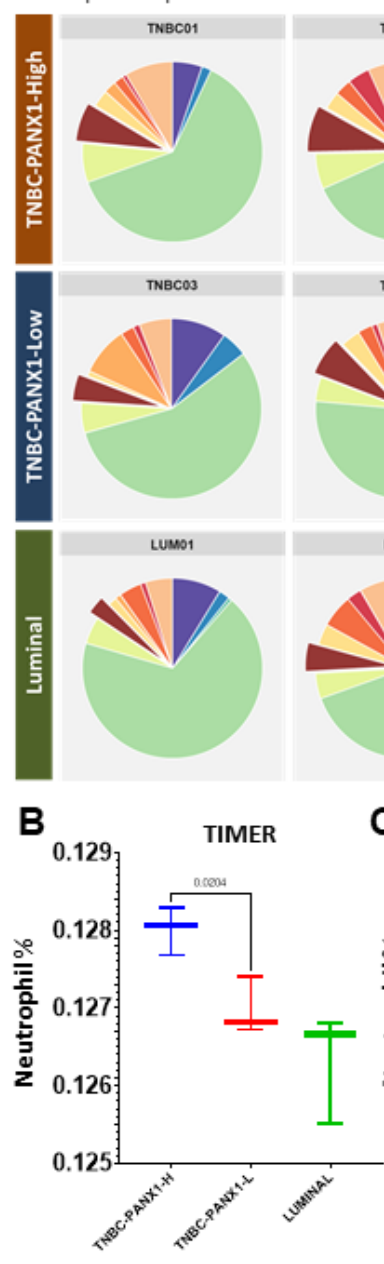
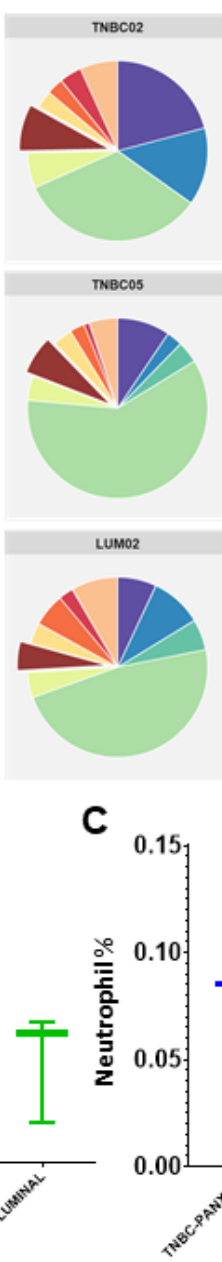

D

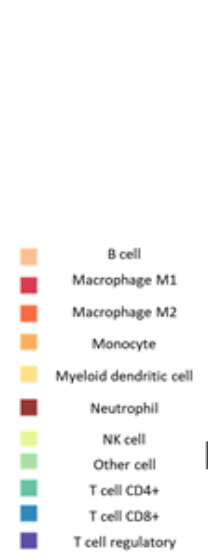

Figure 4
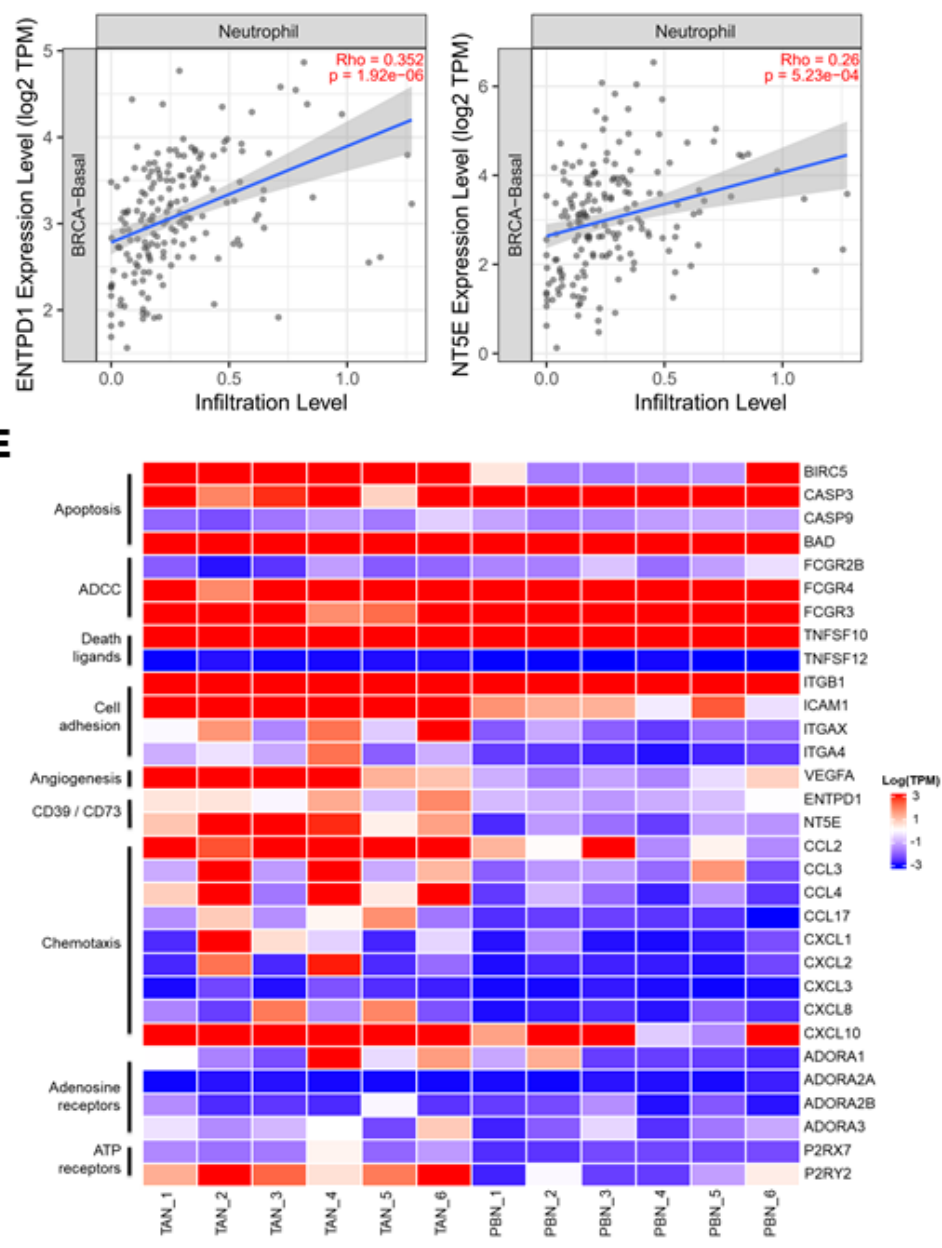

Figure 4

(A) The proportion of infiltrating TANs in Luminal subtype and TNBC (PANX1 high/low expression) surgical specimens; (B) TNBC with high PANX1 expression had more infiltrating TANs than TNBC with low PANX1 expression and the Luminal subtype $(p<0.05$ for TIMER; $p=0.106$ for quanTIseq); (C) TIMER analysis suggested a positive correlation between ENTPD1/NT5E expression and TAN infiltration in the basal-like subtype (TCGA-BRCA data) $(p<0.01)$; (D) Heatmap of the transcriptome analysis of TNBC TANs and PBNs. (TANs, tumor-associated neutrophils; PBNs, peripheral blood neutrophils; TNBC, triplenegative breast cancer) 

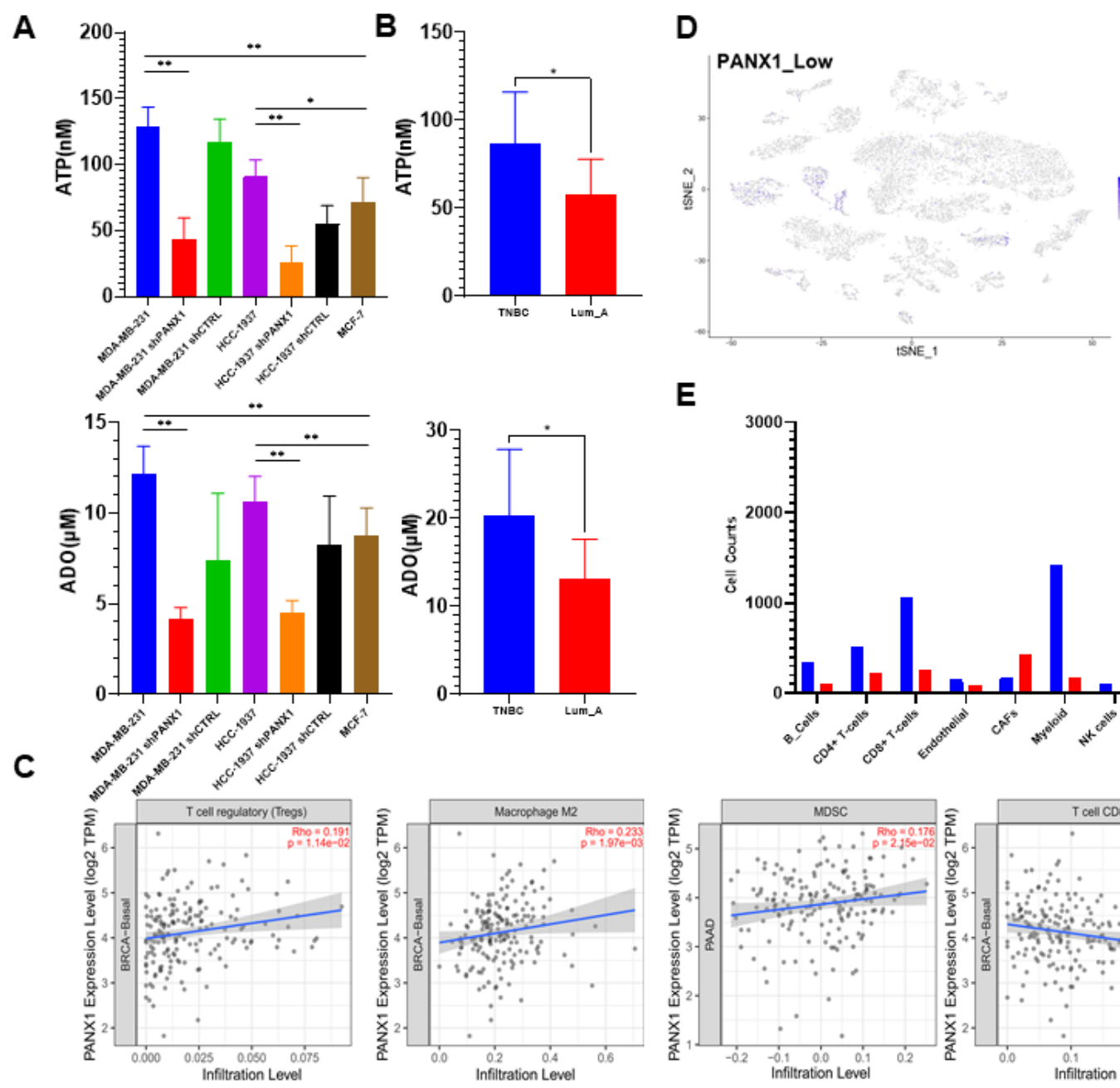

Figure 5

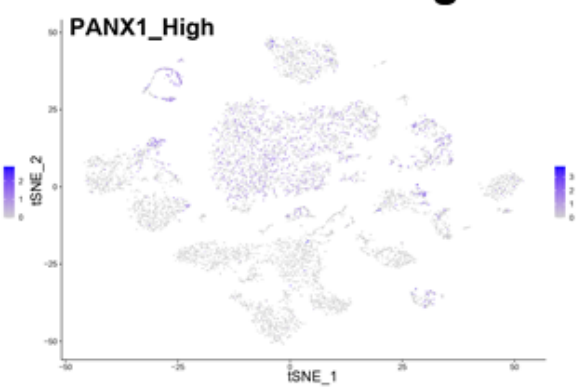

E
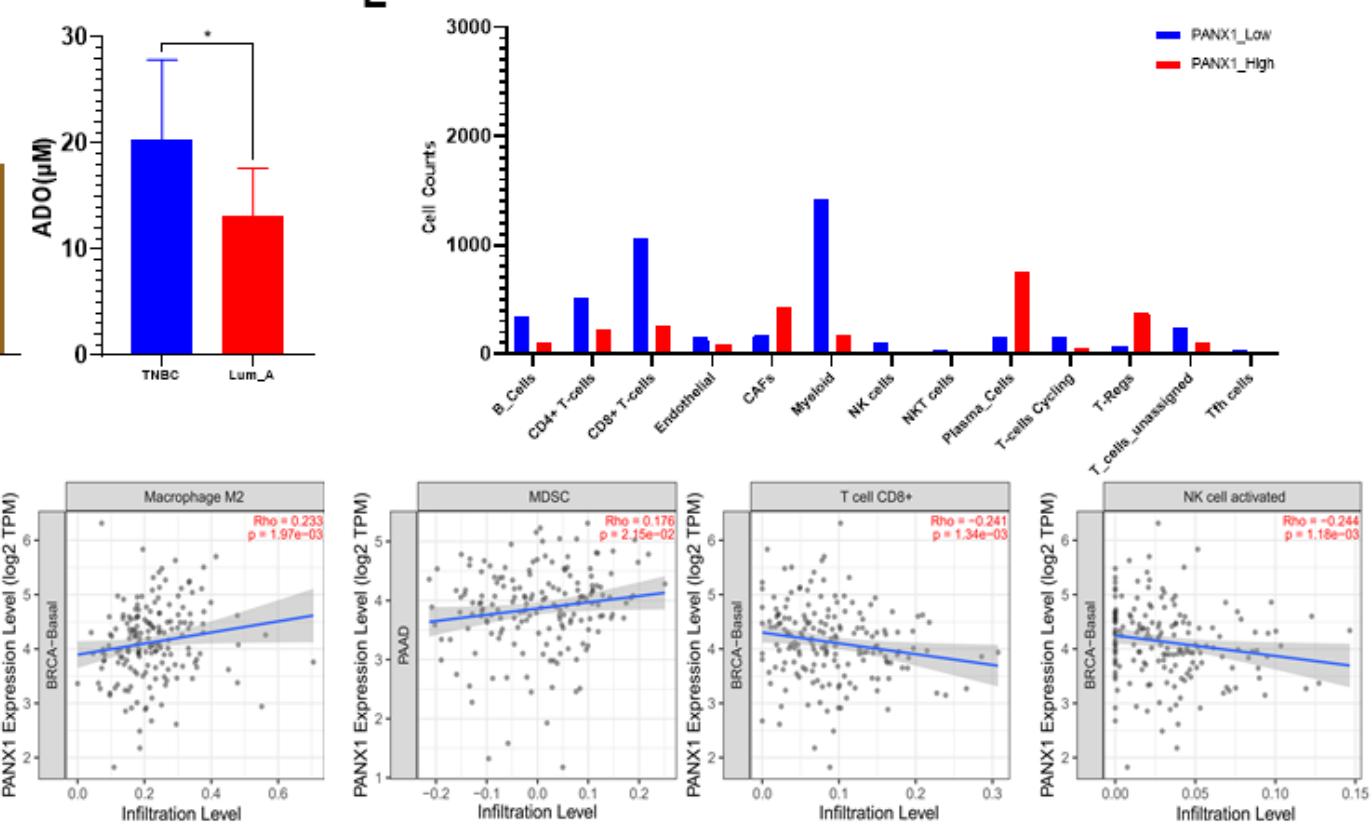

Figure 5

(A) Levels of exATP and exADO in MDA-MB-231, HCC-1937 and MCF-7 cell culture media; PANX1 knock down reduced the levels of exATP and exADO in the supernatant of MDA-MB-231 and HCC-1937 cells; (B) Levels of exATP and exADO in the supernatant of digested tissue from triple-negative and Luminal $A$ breast cancer surgical specimens; (C) The correlation between PANX1 expression and infiltration levels of Tregs, M2-like macrophages, MDSC cells, CD8+ T cells, and NK cells in the TNBC tumor microenvironment by TIMER; (D) The DimPlot of TNBC single-cell transcriptome data in cases with different PANX1 expression levels. (E) The landscape of the tumor microenvironment under different PANX1 expression levels according to TNBC single-cell transcriptome data. Tumor samples with high PANX1 expression had lower infiltration of B cells, CD4+ T cells, CD8+ T cells, myeloid cells, NK cells and NK T cells and higher infiltration of CAFs, plasma cells and Tregs. (CAF, cancer-associated fibroblast; CTRL, control; MDSC, myeloid-derived suppressor cell; NK, natural killer; Treg, regulatory T cell; TNBC, triple-negative breast cancer) $(* p<0.05 ; * \star p<0.01)$ 